EPiC Series in Engineering
Volume 3, 2018, Pages 874-883
HIC 2018. 13th International
Conference on Hydroinformatics

\title{
Optimising Demand Reduction in Water Utilities
}

\author{
Isaac Hamling $^{1}$, William Bloomfield ${ }^{1}$, Kar Yee Dearing ${ }^{1}$, Tim Watson ${ }^{1}$ \\ ${ }^{1}$ ICS Consulting, U.K. \\ isaac@icsconsulting.co.uk
}

\begin{abstract}
Effective water resource planning is an important part of long term strategy for any water company. A good water resource plan will ensure a long-term balance between supply and demand. In this paper we present a scalable, repeatable and transparent model for finding efficient demand reduction solutions given a large number of demand reducing options and small planning regions. This model is being developed for application in UK water utilities for demand side water resource planning to aid results presented to Ofwat, the UK Water regulator, as part of multi-billion-pound long term investment plans. The flexibility and granularity of the approach has been shown to offer significant cost savings while still allowing a utility company to meet customer and stakeholder targets and Ofwat regulatory requirements.
\end{abstract}

\section{Introduction}

Water resource planning is the problem of balancing water supply and demand, in order to have a reliable water supply a company must always have more water available for than is demanded [1]. If the water supplied is lower than customer demand, then some customers will experience supply interruptions. These interruptions carry a heavy penalty making it desirable to ensure sufficient water is available. However, to avoid excess spending on supply it is important to keep supply and demand in a rough equilibrium. This can be done through water supply planning, deciding when to build new water supply tools or upgrade existing ones, or through water demand planning, attempting to reduce water usage. Demand planning attempts to improve water efficiency or the percentage of water supplied going to necessary water usage [2]. A water company's demand consists of leakage, wastage, and usage. Leakage and wastage are both water being 'demanded' with no customer benefit, while usage is customer use, such as drinking and washing, but also includes inefficiency (e.g. leaving taps running while brushing teeth, long showers, watering the garden, etc.).

Water resource planning typically takes place at the water resource region level, where water is considered to be available and movable for free anywhere within that regions water network. A supply, demand balance is done at this region level to determine what demand reductions or supply increases 
are required to maintain a quality of service. In the UK the water industry is regulated by Ofwat [3] who require companies to present expenditure plans for 5-year horizons known as AMPs (Asset Management Plans) [4] where all expenditure must be justified by the benefit to the consumer and society. The sustainability of the water supply is of ever increasing importance to Ofwat and an important part of this sustainability is reducing in water demand. However, reducing this demand in an efficient manner requires a more granular water demand model than the traditional region-based supply demand balance. While in a traditional approach a company may decide to replace pipes in a region to reduce leakage. However, this is not guaranteed to be efficient as some parts of the region may have pipes which can be repaired to give the same reduction in leakage. Therefore, the water region must be split down into smaller sub-regions each with its own unique attributes for consideration and into many different interventions which may include repair and replace.

Traditional water demand models are designed to plan at the water region level where demand can be balanced against supply and build large scale intervention plans for the entire region. We have developed a scalable model for finding the most cost-efficient intervention, sub-region solutions which can meet the demand targets of a water company. This model was created using AIM (Asset Investment Manager) [5] a decision support software package which uses integer programming to solve asset-based models. To meet regulatory requirements, the model provides transparent, repeatable solutions with easy visualisation of the solution.

Our granular model uses integer programming to rapidly find the solution to any set of targets. This means different strategies can be compared for the value they provide, with either the company or regulator able to pick the plan which best suits their needs.

This model is being considered for use by Thames Water for planning demand reductions in future AMPs in the most efficient manner.

\section{Application}

The model developed is built to tactically reduce water demand using a more surgical approach than traditional models. Water demand considers the total water leaving a water network, this must be matched by the water supply entering the network. Water demand consists of three main components, usage, leakage, and wastage. Usage is water being used by customers intentionally. This usage is not $100 \%$ efficient and includes customer inefficiency (E.G. over-washing vegetables). Leakage is water leaking from holes in pipes. Wastage is water used by customers unintentionally (E.G. leaking tap). Water demand is split roughly $75 \%$ usage, $5 \%$ wastage [6], and $20 \%$ leakage for a typical utility company [7] [8]. While it is desirable to have wastage, and leakage as close to zero as possible these can only feasibly be reduced by $50 \%$ [9]. It is estimated that usage can only be reduced by around $15 \%$ of its current level - people are always going to use water [6]. The estimated total available demand reduction is split approximately $47 \%$ usage, $11 \%$ wastage, and $42 \%$ leakage.

To model demand more granularly, the water network is broken down into sub-regions called DMAs (District Metering Areas) [10]. Each DMA has meters and controls which can measure the quantity of water entering and leaving the area and control the pressure of that area. The difference between the water entering and leaving represents the water demand for that DMA. A DMA consists of a set of mains supply pipes, a number of properties either houses, flats, or businesses, communication pipes connecting the properties to the mains, and internal pipes to supply water within the properties. The model has been solved for a problem with around 1,800 DMAs each containing on average approximately 2,500 properties.

The granular model was built to provide solutions for AMP periods which are 5-year time horizons for which UK water utility companies must submit planned work to Ofwat. The model is current being used to plan AMP6 (2020-2024). However, a utility company must also consider the consequences of 
choices over a 40-year time horizon. In addition, this model is utilised to plan AMP7 (2025-2029) and AMP8 (2030-2034) based on the AMP6 plan. Interventions may occur until the end of AMP8 in 2034 while the model calculates effects until 2060. This allows a utility company to generate an AMP6 plan while getting an idea of the required AMP7 and AMP8 spending and interventions alongside the longterm consequences of their choices.

There are many interventions available to reduce the DMA water demand each of which influences at least one the components of demand. These options include metering, water efficiency improvements, mains pipe replacement, Additional ALC (Active leakage control), pressure management, and nonpotable water, these options are taken as examples from Thames Waters WRMP19 (Water Resources Management Plan 2019) [11]. Any of the pipes in this system are capable of leaking but a utility company can only replace and repair pipes they own. All usage, and wastage demand comes from properties. Table 1 shows the demand effect of these interventions and the different options for implementing them.

\begin{tabular}{lllll}
\hline Intervention & Usage & Wastage & Leakage & Options \\
\hline Metering & Y & Y & Y & Household, non-household, flat, bulk \\
Water efficiency & Y & Y & & SHV, SBV, wastage fix \\
Mains replacement & & & Y & Replacement: 25. 50, 75, 100\% \\
$\begin{array}{l}\text { Additional ALC } \\
\text { Pressure }\end{array}$ & & & Y & Valves, washout, find and fix \\
management & & & Y & Pressure management schemes \\
Non-potable water & Y & & & Rainwater collection, Greywater recycling \\
\hline
\end{tabular}

Table 1 Effect on demand of each available demand intervention

\subsection{Metering}

Metering is the process of placing a smart meter on a property or group of properties which measures the water demanded by that property.

Meters can be either placed onto a house or business, onto an apartment block (bulk metering), or onto all individual apartments. The more meters are the placed the easier it is to locate leaks in pipes (I.E. if all properties are metered then the leakage in supply pipes can be exactly measured as DMA demand minus metered demand).

Additionally, by metering a property the customer becomes more aware of their usage and as such may reduce their usage. If the metered demand is especially high, it may lead to the discovery of wastage or leakage within the property. In particular bulk metering leads to leakage reduction as the company can better locate leaks within their network.

A company can also charge metered properties per ML of water used which can encourage further usage reduction.

\subsection{Water efficiency}

Water efficiency is the process of teaching customers how to more efficiently use water. This is applied in the form of SHVs (Smarter Home Visits) and SBVs (Smarter Business Visits) where workers teach customers how to be water efficient and provide leaflets and water efficiency devices. This process provides an immediate reduction in usage, and wastage which diminishes over time as customers forget the water efficiency lessons. For this reason, once a SHV or SBV is conducted followups are done every few years.

The effectiveness of a SHV is dependent on whether a property is metered. If a property is metered, then the customer can get immediate feedback on the effectiveness of usage reductions increasing the effectiveness of the SHV. 
Other water efficiency interventions include wastage fix, and housing association fix. Wastage fix involves sending someone to fix wastage problems on properties.

\subsection{Mains replacement}

Mains replacement is the replacement of an entire pipe in the water supply network, which removes most of the leakage form the pipe.

All pipe replacements are considered by percentage of the DMA being replaced, at either $25 \%, 50 \%$, $75 \%$ or $100 \%$ replacement. Leak rates and replacement cost are calculated in a separate pipe level model and then ranked to form the most to least beneficial $25 \%$ bins that are then fed into the IDM model. Replacing pipes reduces the effectiveness of pressure management and DMA enhancement as there are less leaks to find or mitigate.

\subsection{Additional ALC}

Additional ALC is a program above and beyond the standard detection and repair work conducted to find and fix leaks in a DMA. The network is reconfigured within the DMA to allow easier detection of leakage, by installing new valves, and washouts, or enabling access to previously difficult to access valves. This allows increased find and fix activity and reduced leakage.

\subsection{Pressure management}

Pressure management alters the pressure in a DMA to reduce the overall leakage within that DMA. This can be improved through the installation of new pressure management schemes within a DMA. These may allow better control of the pressure within a DMA or employ new strategies in a DMA on how to leverage pressure management.

\subsection{Non-potable water}

Non-potable water is the supplement of mains water with non-potable water which is sourced from an area around a group of DMAs. This water can be used as toilet water, watering gardens, etc. Supplying non-potable water reduces main usage by the quantity of water used in the activity it replaces. As this is intervention affects a group of DMAs rather than a single DMA it is difficult to model if it is necessary to specific its exact timing between other interventions which may not match between DMAs.

\section{Methodology}

Ofwat requires any solution be thoroughly verified and validated before being put into place for any decision making. The solution must also be repeatable and transparent, so the regulator can audit any solutions provided. For this reason, we choose to use AIM an asset level modelling package with visualisation and reporting designed to meet these regulations. AIM utilises an integer program which can guarantee optimality and as such for any consistently formulated problem will always provide an identical solution.

The model is built for around 1,800 sub-regions (DMAs), with a 40-year time horizon, 15 decision periods, and 16 different intervention options. Depending on how these are combined there are between 400,000 options if only one intervention is chosen per DMA and $\mathbf{3 \times 1 0 ^ { 1 6 }}$ if all intervention permutations are considered. 


\subsection{Integer Program}

The software package AIM [5] was used to build the integer programming model for the water demand, and reductions. AIM is an asset modelling tool which provides a structured approach to building an integer program to find the optimal asset intervention strategy. AIM does this by computing reactive (or baseline doing nothing) positions for the DMAs that are then compared to proactive (intervention) positions to calculate the benefit of those choices.

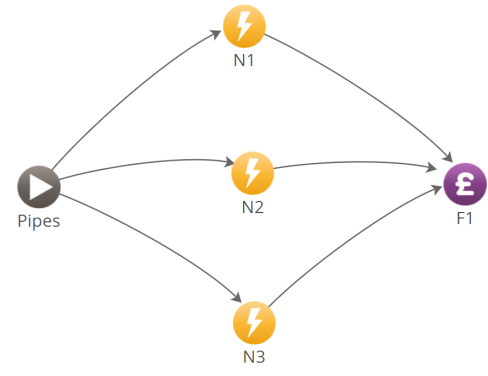

Figure 1: Example risk map structure

The problems are formulated using a risk map which provides a graphical version of the complex equations used to compute demand values. Figure 1 shows a simple example risk map with three initiating from the source node which then combine into a final cost node. The equation represented by this risk map is $(\mathrm{N} 1+\mathrm{N} 2+\mathrm{N} 3)^{*} \mathrm{~F} 1$. Each node contains a baseline equation for the model and a post intervention equation if an intervention affects the node. For example:

$$
N 1=\left\{\begin{array}{c}
1.5 \cdot \text { NumProperties when Reactive } \\
1 \cdot \text { NumProperties when Metered }
\end{array}\right.
$$

AIM then builds an IP (integer program) which trades off the cost of an intervention against the benefits received. The variables in this IP consist of one variable for each DMA, for each intervention time period, and each intervention option. This IP is formulated as standard binary IP [12] and solved using Gurobi [13].

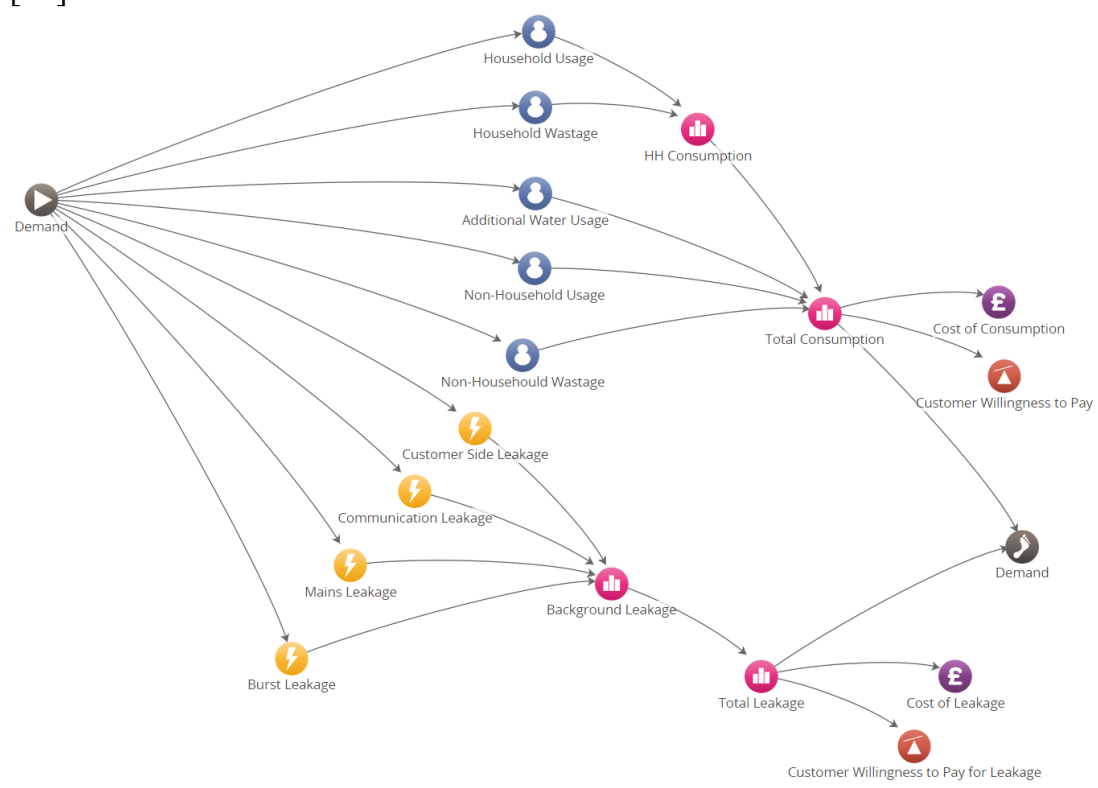

Figure 2: Risk map for demand model 
In standard configuration AIM only allows one intervention to be chosen on an asset for the entire time period. However, it is possible to create new interventions which are combinations of different interventions over time called strategy trains. Strategy trains offer the ability to code business rules and dependent relationships between different interventions. Alternatively, if the interventions have constant proactive effects independent of the other interventions they can be considered separately as superimposed DMA intervention pairs. This superposition offers greater flexibility in the choices you can make regarding timing between different interventions.

Figure 2 shows the risk map for the demand model with nodes representing each demand type. The map consists of nodes for; household usage, and wastage; non-household usage, and wastage; and customer, communication, mains and burst leakage. These nodes have reactive models, and proactive models for different interventions depending on the impact they have on demand. The nodes then combine to consumption (usage and wastage) and leakage. Consumption and leakage have associated cost and give total demand.

\subsection{Demand Model}

\subsubsection{Strategy Train}

The strategy train model is a form of manual column generation for the AIM integer program. These strategy trains allow users to add specific intervention combinations called strategy trains. E.G. a strategy train could be created where metering is done on a DMA followed by a smarter home visit 2 years later. Figure 3 shows example strategy trains between the intervention options; metering, SHV, replacement, and enhancement. This model is powerful if there are a low number of interventions or if interventions come in specific and consistent combinations for business reasons. For example, SHV get repeated every few years and are enhanced by metering, so it is likely there is a consistent combination of SHVs and metering which the business would want to include. However, this model has more than 16 interventions many of which are independent with each affected a different type of demand. In order to account for the potential combinations over 200 strategy trains were developed for the 15 -year period with interventions. Even with such a large quantity the unrelated nature of the interventions meant that after the first 5 years the model would lack the flexibility to make good intervention choices.

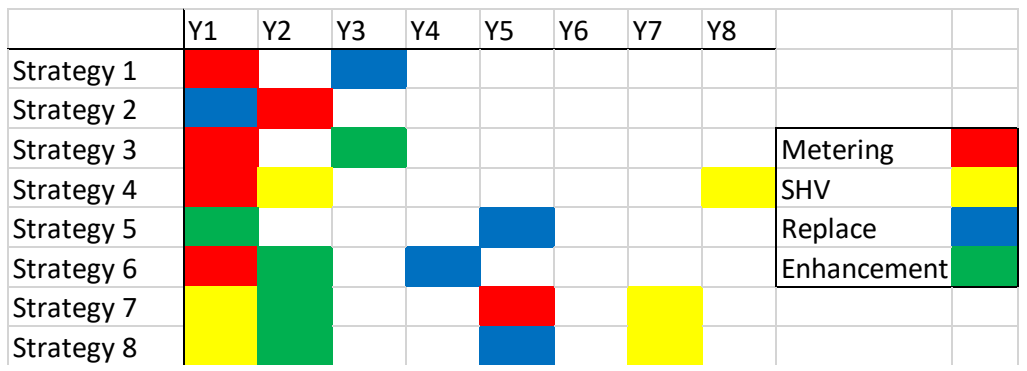

Figure 3: Gantt chart of example strategy trains from year of first intervention

\subsubsection{Superposition}

Due to the lack of flexibility within the strategy train model and the independent nature of many of the interventions a new modelling approach was needed. Finding efficient solutions for demand requires a model able to independently consider the value of mains replacement and metering or pressure management. The new integer program takes the interventions superimposes them onto the DMAs themselves creating a set of DMA, intervention pairs allowing each intervention to be selected independently. 
This change significantly increases the number of decisions available to the integer program but because the interventions are independent the event orders don't alter the preferences of other interventions. In fact, this updated model suffered minimally in terms of increased times to optimality.

\section{Results}

All models were solved using ICS utilities testing network for example planning scenarios. These scenarios include leakage reduction targets, demand reduction targets, and meter installation targets. All models are run with an IP objective function to minimise spending on interventions.

The ICS utilities dataset consists of 1,800 DMAs with 17 interventions for the 15-year decision horizon. The models calculate pipe leakage and deterioration as well as household and non-household usage and wastage. Overall calculating the data inputs for the models takes around 10 minutes while the models take 10 minutes to solve.

Overall performance of the traditional, strategy train, and superposition models are compared in table 2. Overall the superposition model can reduce the expected budget to $80 \%$ of the strategy train budget. Figure 4 shows the cumulative cost of the strategy train and superposition models over the planning horizon for the same targets. The superposition is overall cheaper, and the difference diverges as the strategy train model runs out of long term options.

\begin{tabular}{lll}
\hline Model & Percentage of budgeted cost & Solution Time \\
\hline Strategy Train & $100 \%$ & $10 \mathrm{mins}$ \\
Superposition & $80 \%$ & $10 \mathrm{mins}$ \\
\hline
\end{tabular}

Table 2 Model performance in terms of cost and solution speed

Figure 5 shows the demand savings over the planning horizon, these savings are split by intervention type, and demand category. Metering interventions have the biggest impact followed by replacement, and water efficiency. The interventions are split according to the savings in table 1.

Figure 6 shows a spatial view of demand savings over 10 years, each dot represents a DMA, the size and colour of the dot show its relative contribution to leakage savings. This shows a significant difference in contribution to savings between DMAs.

\section{Discussion}

The results of the models show an improvement in solution quality between the strategy train and superposition model, by meeting the same targets at a lower cost. Figure 6 shows how the break down at DMA level gives the models the ability to target demand reductions in areas with higher net benefits, as the potential savings are not evenly distributed.

The influence of different interventions is shown in figure 5. While metering offers the largest savings over multiple categories there are also significant contributions from mains replacement, additional ALC, and water efficiency. By allowing metering and replacement to be independently selected the superposition model can maximise savings from both without restricting future choices in water efficiency or additional ALC.

The low run times of the model allow testing of a wide variety of targets and sensitivity analysis to determine the value of future targets and spending. This is especially powerful over the traditional model where only one solution was generated. 


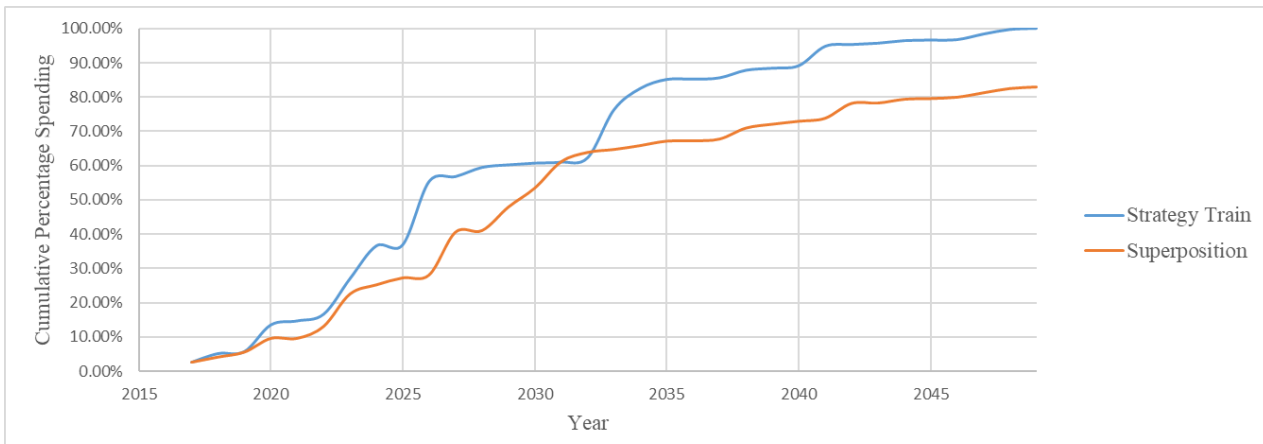

Figure 4: Relative cost over time comparing strategy train and superposition model

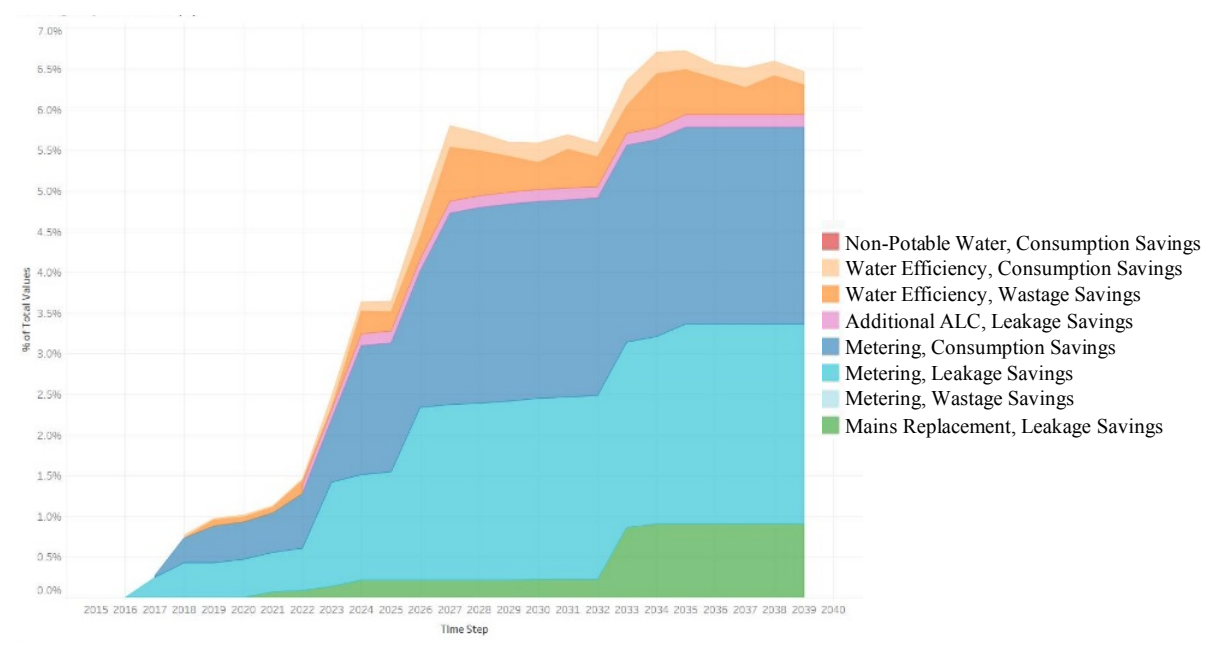

Figure 5: Demand savings by intervention groups and demand type

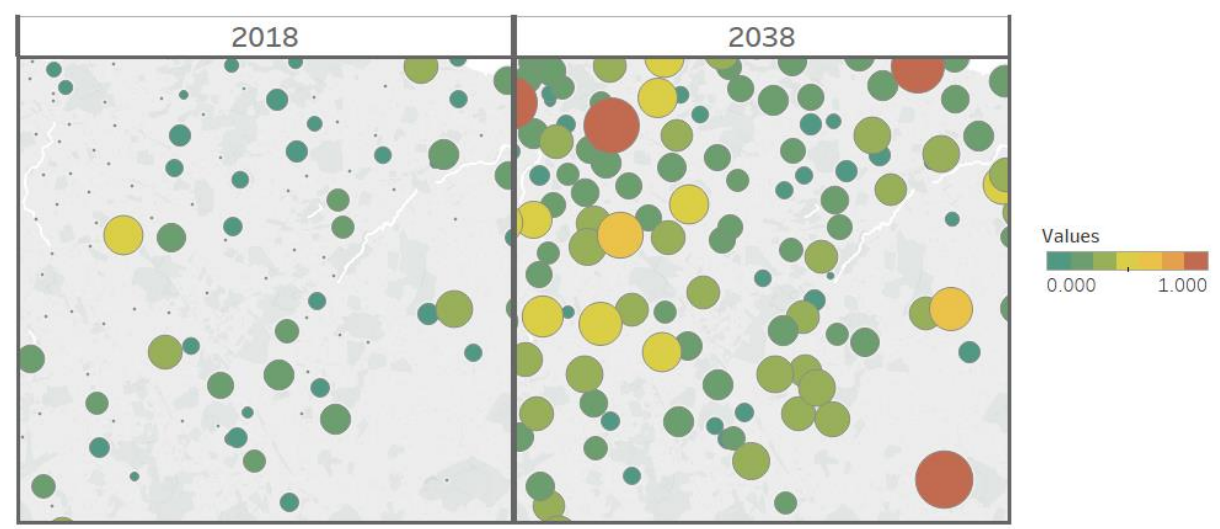

Figure 6: Spatial view of water savings in DMAs over 10 years 


\section{Conclusion}

Overall these demand models provide significantly increased flexibility over the existing standard and can rapidly provide solutions to different scenarios. This allows a water utility company to test many different targets to see how much it would cost and what interventions are necessary to meet the targets.

These models are especially useful improving the environmental sustainability of water demand into the future as they allow companies to understand the costs and benefits of different interventions and how they trade-off against one another. This is especially important for meeting Ofwat regulatory requirements. Ofwat is also able to see the trade-offs from different scenarios and targets and the modelling functions and outputs are easily auditable.

Results show the effect of sub-regions and intervention breakdowns. With the savings being unevenly spread throughout DMAs rather than over an entire region and savings being unevenly distributed between different interventions. This more flexible model is currently being implemented at Thames water but requires verification before final implementation, and result can be presented.

\section{References}

[1] V. B. E. Loucks DP, Water resource systems planning and management: An introduction to methods, models, and applications., Springer, 2017.

[2] Baumann, D. Duane, J. J. Boland and M. W. Hanemann, "Urban water demand management and planning," McGraw-Hill Professional, 1997.

[3] The Water Services Regulation Authority, "Ofwat," [Online]. Available: https://www.ofwat.gov.uk/. [Accessed 2404 2018].

[4] Drinking Water Inspectorate , "Price Review Process - DWI, UK," [Online]. Available: http://www.dwi.gov.uk/stakeholders/price-review-process/index.htm. [Accessed 04 2018].

[5] Probit Consulting, "Asset Investment Manager by Probit Consulting," [Online]. Available: https://www.probitconsulting.com/. [Accessed 2404 2018].

[6] Waterwise, "Water Efficiency Strategy for the UK," Waterwise, 2017.

[7] Anglian Water Business, "UK businesses leak thousands of pounds in wasted water Anglian Water Business," Anglian Water Business, 3011 2017. [Online]. Available: https://www.anglianwaterbusiness.co.uk/uk-businesses-leak-thousands-pounds-wastedwater/. [Accessed 2004 2018].

[8] D. Carrington, "Water companies losing vast amounts through leakage, as drought fears rise | Environment | The Guardian," The Guardian, 1105 2017. [Online]. Available: https://www.theguardian.com/environment/2017/may/11/water-companies-losing-vastamounts-through-leakage-raising-drought-fears. [Accessed 04 2018].

[9] A. Johnson and J. Burton, "Water torture: 3,300,000,000 litres are lost every single day through leakage | The Independent," The Independent, 2407 2010. [Online]. Available: https://www.independent.co.uk/news/uk/home-news/water-torture-3300000000-litres-arelost-every-single-day-through-leakage-2034999.html. [Accessed 04 2018].

[10] F. M, "Leakage management and control: a best practice training manual," World Health Oragnisation, 2001. 
[11] Thames Water, "Appraisal of demand options," in Water Resources Management Plan 2019, Reading, Thames Water, 2017, p. Section 8.

[12] A. Schrijver, Theory of linear and integer programming, John Wiley \& Sons, 1998.

[13] Gurobi Optimization, "Gurobi Optimization - The State-of-the-Art Mathematical Programming Solver," [Online]. Available: http://www.gurobi.com/. [Accessed 2404 2018].

[14] A. Voronkov, "EasyChair conference system," 2004. [Online]. Available: easychair.org.

[15] D. Carlisle, "graphicx: Enhanced support for graphics.," April 2010. [Online]. Available: http://www.ctan.org/tex-archive/ help/Catalogue/entries/graphicx.html.

[16] Wikipedia, "EasyChair," [Online]. Available: https://en.wikipedia.org/wiki/EasyChair.

[17] A. Voronkov and K. Hoder, "Templates," EasyChair, [Online]. Available: https://easychair.org/proceedings/template.cgi?a=12732737.

[18] A. Voronkov, "Keynote talk: EasyChair," in Proceeedings of the 29th ACM/IEEE International Conference on Automated Software Engineering, ACM, 2014, pp. 3-4. 